\title{
Correction to: On Traveling Solitary Waves and Absence of Small Data Scattering for Nonlinear Half-Wave Equations
}

\author{
Jacopo Bellazzini ${ }^{1}$ [D, Vladimir Georgiev ${ }^{2,3,4}$, Enno Lenzmann ${ }^{5}$, Nicola Visciglia ${ }^{2}$ \\ ${ }^{1}$ Università di Sassari, Via Piandanna 4, 70100 Sassari, Italy. E-mail: jbellazzini@ uniss.it \\ 2 Dipartimento di Matematica, Università di Pisa, Largo B. Pontecorvo 5, 56100 Pisa, Italy. \\ E-mail: georgiev@dm.unipi.it; viscigli@dm.unipi.it \\ 3 Faculty of Science and Engineering, Waseda University, 3-4-1, Okubo, Shinjuku-ku, Tokyo 169-8555, Japan \\ 4 IMI-BAS, Acad. Georgi Bonchev Str., Block 8, 1113 Sofia, Bulgaria \\ 5 Departement Mathematik und Informatik, Universität Basel, Spiegelgasse 1, 4051 Basel, Switzerland. \\ E-mail: enno.lenzmann@unibas.ch
}

Published online: 24 March 2021 - @ S Springer-Verlag GmbH Germany, part of Springer Nature 2021

\section{Correction to: Commun. Math. Phys. 372, 713-732 (2019) https://doi.org/10.1007/s00220-019-03374-y}

In this erratum, we correct an error in Lemma 3.1 in our paper [1]. Although the main results in [1] are ultimately not affected by this correction, we deem it necessary to provide the adequate corrections here. We are grateful to Jie Chen for drawing our attention to this matter and for sharing his insights in [2].

We use the notation from [1] and we recall that the optimal constant for GagliardoNirenberg type inequality $\left(\mathrm{GNS}_{v}\right)$ is given by

$$
\mathbf{C}_{v, d, p}=\sup _{u \in H^{1 / 2}\left(\mathbb{R}^{d}\right) \backslash\{0\}} \frac{\|u\|_{L^{p+1}}^{p+1}}{\left\|(|\xi|-v \cdot \xi)^{1 / 2} \widehat{u}\right\|_{L_{\xi}^{2}}^{d(p-1)}\|u\|_{L^{2}}^{p+1-d(p-1)}},
$$

where $d \geqslant 1, v \in \mathbb{R}^{d}$ with $|v|<1$, and $1<p<p_{*}$ with $p_{*}=+\infty$ when $d=1$ and $p_{*}=\frac{d+1}{d-1}$ when $d \geqslant 2$.

In Lemma 3.1 in [1], it was stated that

$$
\mathbf{C}_{v, d, p} \sim(1-|v|)^{-\frac{d(p-1)}{2}} .
$$

However, the claimed estimate (2) is wrong except for dimension $d=1$. The corrected version of Lemma 3.1 reads as follows. 
Lemma 1. For $d \geqslant 1,1<p<p_{*}$, and $|v|<1$, we have the estimate

$$
\mathbf{C}_{v, d, p} \sim(1-|v|)^{-\frac{(d+1)(p-1)}{4}} .
$$

Proof of Lemma 1. It remains to treat the case $d \geqslant 2$, since the proof in [1] is correct for dimension $d=1$. Without loss of generality, we can assume that $v=|v| e_{1}=$ $(|v|, 0, \ldots, 0) \in \mathbb{R}^{d}$ with $|v|<1$.

For $x, \xi \in \mathbb{R}^{d}$, we write $x=\left(x_{1}, x^{\prime}\right) \in \mathbb{R} \times \mathbb{R}^{d-1}$ and $\xi=\left(\xi_{1}, \xi^{\prime}\right) \in \mathbb{R} \times \mathbb{R}^{d-1}$. For any $\mu>0$ and $u \in H^{1 / 2}\left(\mathbb{R}^{d}\right)$ with $u \not \equiv 0$, we use the rescaling in $x_{1}$ given by

$$
u_{\mu}(x)=\mu^{1 / 2} u\left(\mu x_{1}, x^{\prime}\right),
$$

which preserves the $L^{2}\left(\mathbb{R}^{d}\right)$-norm $\|u\|_{L^{2}}=\left\|u_{\mu}\right\|_{L^{2}}$. Then we have

$$
\begin{gathered}
\frac{\left\|u_{\mu}\right\|_{L^{p+1}}^{p+1}}{\left\|(|\xi|-v \cdot \xi)^{1 / 2} \widehat{u}_{\mu}\right\|_{L_{\xi}^{2}}^{(p-1)}\left\|u_{\mu}\right\|_{L^{2}}^{p+1-d(p-1)}} \\
=\frac{\mu^{(p-1) / 2}\|u\|_{L^{p+1}}^{p+1}}{\left\|P_{v, \mu}(\xi)^{1 / 2} \widehat{u}\right\|_{L_{\xi}^{2}}^{d(p-1)}\|u\|_{L^{2}}^{p+1-d(p-1)}},
\end{gathered}
$$

where

$$
P_{v, \mu}\left(\xi_{1}, \xi^{\prime}\right)=\sqrt{\mu^{2} \xi_{1}^{2}+\left|\xi^{\prime}\right|^{2}}-|v| \mu \xi_{1}
$$

The claimed estimate in Lemma 1 will easily follow if we choose

$$
\mu=\frac{1}{\sqrt{1-|v|}} \geqslant 1
$$

together with the following estimates

$$
\begin{aligned}
& P_{v, \mu}\left(\xi_{1}, \xi^{\prime}\right) \lesssim \sqrt{1-|v|}|\xi| \text { for } \xi_{1}>0 \text { and }\left|\xi^{\prime}\right| \leqslant \xi_{1}, \\
& P_{v, \mu}\left(\xi_{1}, \xi^{\prime}\right) \gtrsim \sqrt{1-|v|}|\xi| \text { for } \xi=\left(\xi_{1}, \xi^{\prime}\right) \in \mathbb{R}^{d} .
\end{aligned}
$$

Indeed, to prove the lower bound in Lemma 1 , we take $u \in H^{1 / 2}\left(\mathbb{R}^{d}\right), u \not \equiv 0$, such that $\operatorname{supp} \widehat{u}(\xi) \subseteq\left\{\left|\xi^{\prime}\right| \leqslant \xi_{1}\right\}$. Then we can apply the estimate (5) in combination with (3) and (4) to obtain the lower bound

$$
\mathbf{C}_{v, d, p} \gtrsim \frac{(1-|v|)^{-\frac{(p-1)}{4}}\|u\|_{L^{p+1}}^{p+1}}{(1-|v|)^{\frac{d(p-1)}{4}}\left\||\xi|^{1 / 2} \widehat{u}\right\|_{L_{\xi}^{2}}^{d(p-1)}\|u\|_{L^{2}}^{p+1-d(p-1)}} \gtrsim(1-|v|)^{-\frac{(d+1)(p-1)}{4}} .
$$

On the other hand, if we apply estimate (6) with (4), we deduce the upper bound

$$
\mathbf{C}_{v, d, p} \lesssim \frac{(1-|v|)^{-\frac{(p-1)}{4}}\|u\|_{L^{p+1}}^{p+1}}{(1-|v|)^{\frac{d(p-1)}{4}}\left\||\xi|^{1 / 2} \widehat{u}\right\|_{L_{\xi}^{2}}^{d(p-1)}\|u\|_{L^{2}}^{p+1-d(p-1)}} \lesssim(1-|v|)^{-\frac{(d+1)(p-1)}{4}}
$$


Hence it remains to prove (5) and (6). To get the upper bound (5) of the symbol $P_{v, \mu}$, we set $a=\mu \xi_{1}, b=\left|\xi^{\prime}\right| \geqslant 0$ and assuming $a=\mu \xi_{1}>0,\left|\xi^{\prime}\right| \leqslant \xi_{1}$ we can write

$$
P_{v, \mu}\left(\xi_{1}, \xi^{\prime}\right)=\frac{b^{2}}{\sqrt{a^{2}+b^{2}}+a}+(1-|v|) a \lesssim \frac{b^{2}}{a}+(1-|v|) a \lesssim \sqrt{1-|v|}|\xi| .
$$

This shows (5).

In a similar way and assuming that $a=\mu \xi_{1}>0$ and $b=\left|\xi^{\prime}\right|$, we have the lower bound as follows

$$
P_{v, \mu}\left(\xi_{1}, \xi^{\prime}\right)=\frac{b^{2}}{\sqrt{a^{2}+b^{2}}+a}+(1-|v|) a \geqslant \frac{b^{2}}{2(a+b)}+(1-|v|) a .
$$

If $\xi_{1} \geqslant\left|\xi^{\prime}\right|$, then we have $|\xi| \sim \xi_{1}$ and we find

$$
P_{v, \mu}\left(\xi_{1}, \xi^{\prime}\right) \geqslant \frac{b^{2}}{2 a+2 b}+(1-|v|) a \geqslant(1-|v|) a \sim(1-|v|) \mu|\xi| .
$$

If $\xi_{1} \leqslant\left|\xi^{\prime}\right|$, then we have $|\xi| \sim\left|\xi^{\prime}\right|$ and with $\mu \geqslant 1$ we can write

$$
P_{v, \mu}\left(\xi_{1}, \xi^{\prime}\right) \geqslant \frac{b^{2}}{2(a+b)}+(1-|v|) a \geqslant \frac{b^{2}}{2(a+b)} \geqslant \frac{\left|\xi^{\prime}\right|}{2(\mu+1)} \sim \frac{1}{\mu}|\xi| .
$$

Thus, for any $a=\mu \xi_{1}>0$, we have found that

$$
P_{v, \mu}\left(\xi_{1}, \xi^{\prime}\right) \gtrsim \min \left((1-|v|) \mu, \frac{1}{\mu}\right)|\xi| \text {. }
$$

Recalling (4), we obtain (6) for $\xi_{1}>0$. Since for $\xi_{1} \leqslant 0$ we have stronger inequality

$$
P_{v, \mu}\left(\xi_{1}, \xi^{\prime}\right) \gtrsim \sqrt{a^{2}+b^{2}} \gtrsim|\xi|,
$$

we can conclude that (6) is valid for all $\xi=\left(\xi_{1}, \xi^{\prime}\right) \in \mathbb{R}^{d}$.

The proof of Lemma 1 is now complete.

The corrected upper bound for $\mathbf{C}_{v, d, p}$ in Lemma 1 above has now to be taken into account in various statements in [1] (without affecting the main results a posteriori). In particular, the bounds in Lemma 3.2 in [1] have to be changed into

$$
\left\|Q_{v}\right\|_{L^{2}}^{2} \lesssim(1-|v|)^{\frac{d+1}{2}} \text { and }\left\|Q_{v}\right\|_{\dot{H}^{1 / 2}}^{2} \lesssim(1-|v|)^{\frac{d-1}{2}} .
$$

Likewise, the main estimate in the proof of Theorem 1.2 for $d \geqslant 2$ has to be changed into

$$
\left\|Q_{v}\right\|_{H^{1 / 2}}^{2}=\left\|Q_{v}\right\|_{L^{2}}^{2}+\left\|Q_{v}\right\|_{\dot{H}^{1 / 2}}^{2} \lesssim(1-|v|)^{\frac{d+1}{2}}+(1-|v|)^{\frac{d-1}{2}}<\varepsilon^{2},
$$

which is still valid. Finally, we remark that estimates (B.4) and (B.5) in the proof of Theorem 1.3 must be changed into

$$
\mathbf{C}_{v, d, p_{*}} \lesssim(1-|v|)^{-\frac{1}{2} \frac{d+1}{d-1}} \text { and }\left\|W_{v}\right\|_{\dot{H}^{1 / 2}}^{2} \lesssim(1-|v|)^{\frac{d-1}{2}} .
$$

The rest of the proof of Theorem 1.3 follows in same manner as given in [1].

Publisher's Note Springer Nature remains neutral with regard to jurisdictional claims in published maps and institutional affiliations. 


\section{References}

1. Bellazzini, J., Georgiev, V., Lenzmann, E., Visciglia, N.: On traveling solitary waves and absence of small data scattering for nonlinear half-wave equations. Commun. Math. Phys. 372(2), 713-732 (2019). https:// doi.org/10.1007/s00220-019-03374-y

2. Chen, J.: Private communication 